\title{
Tripanosomiasis americana, una mirada desde el tratamiento
}

\author{
American trypanosomiasis, a new look at its treatment
}

\author{
Emmanuel Sánchez¹, María Camila Vélez¹, Marcos Restrepo", \\ Juan Sebastián Marín', Daniel Gallego' \\ 'Estudiantes de medicina. Grupo de Investigación de Biología de Sistemas; Facultad de Medicina; Universidad Pontificia Bolivariana, Sede
}

Central Medellín, Circular 1 No. 70-01, Medellin, Colombia.

\begin{abstract}
Resumen
La tripanosomiasis americana o enfermedad de Chagas es una infección parasitaria causada por el parásito flagelado Trypanosoma cruzi, cuyo principal vector es un insecto de la clase hemíptera conocido como triatomino. La quimioterapia, adicional a un control oportuno de vectores y rigurosidad en el control de las transfusiones, son los elementos más importantes para el manejo de esta parasitosis. En la actualidad, el mercado farmacéutico solo ofrece nifurtimox y benznidazol como opciones terapéuticas, y a pesar de que se han obtenido resultados satisfactorios con el uso de estos medicamentos en fases agudas de la enfermedad, tripanosomiasis congénita y accidentes de laboratorio, la efectividad en las fases crónicas es notoriamente menor. Además, ambos fármacos generan ciertos efectos adversos que deben ser tenidos en cuenta por el personal de la salud, antes de iniciar el debido manejo de esta enfermedad. El objetivo del presente artículo es revisar el estado actual del tratamiento farmacológico de la tripanosomiasis americana, buscando resumir los nuevos avances terapéuticos y dar a conocer las limitaciones de los mismos debido a sus efectos adversos.

Palabras clave. Enfermedad de Chagas; Tripanosomiasis; Terapéutica.
\end{abstract}

Abstract

American trypanosomiasis or Chagas disease is a parasitic infection caused by the flagellate parasite Trypanosoma cruzi, whose main vector is an insect of the Hemiptera class, called triatomine. Chemotherapy, in addition to an appropriate vector control and a rigorous control of transfusions, are the most important strategies for the management of this parasitosis. Currently, the pharmaceutical market only offers nifurtimox and benznidazole as treatment options, and although satisfactory results have been obtained with the use of these drugs in either acute stages of the disease, congenital trypanosomiasis and laboratory accidents, its effectiveness in the chronic phases is significantly smaller. In addition, both drugs produce some side effects that must be taken into account by health workers, before starting the proper management of the disease. The aim of this article is to review the current state of the American trypanosomiasis treatment, in order to summarize the new advances in therapeutics and to introduce their limitations due to adverse effects.

Keywords. Chagas Disease; Trypanosomiasis; Therapeutics.

An Fac med. 2016;77(1):39-44 / http://dx.doi.org/10.15381/anales.v77i1.11551

\section{INTRODUCCIÓN}

La tripanosomiasis americana, también conocida como enfermedad de Chagas, es la infección parasitaria más importante en las Américas, en donde afecta de 8 a 10 millones de personas ${ }^{(1-3)}$. Es causada por el parásito flagelado Trypanosoma cruzi, cuyo principal vector es un insecto de la clase hemíptera conocido como triatomino. El parási- to también puede ser adquirido por el consumo de alimentos y bebidas contaminadas, trasplantes de órganos, transfusiones sanguíneas y por vía congénita, siendo estas vías menos frecuentes ${ }^{(1,4)}$.

En el curso natural de la enfermedad es posible diferenciar tres fases clínicas: una fase aguda, usualmente asintomática, que tiene lugar de 1 a 3 semanas después de la infección, en donde los pacientes tienen un pronóstico benig- no y los síntomas desaparecen después de 1 a 2 meses; una fase indeterminada, en la que no se presentan manifestaciones sintomáticas y, a pesar de que la parasitemia no alcanza los niveles de la fase aguda, el paciente constituye una importante fuente de infección; y la fase crónica, cuya aparición tiene lugar décadas después de las manifestaciones agudas y lleva a condiciones clínicas como cardiomiopatía, arritmias ven- 
triculares, muerte súbita, megaesófago, megacolon y compromiso del sistema nervioso periférico ${ }^{(5-7)}$.

Una de las manifestaciones clínicas más importantes en los adultos es la miocardiopatía infecciosa, llegando a ser la tripanosomiasis americana la principal causa de esta condición a nivel mundial; mientras que en los niños es frecuente la aparición de miocarditis y meningoencefalitis ${ }^{(8)}$. Se estima que alrededor de 10 mil personas mueren anualmente debido a las manifestaciones crónicas de la enfermedad de Chagas, siendo las alteraciones cardiacas y gastrointestinales las principales causas de muerte ${ }^{(7,9)}$.

Los factores de riesgo para el desarrollo de esta condición han sido ampliamente establecidos, entre ellos, residir o incluso proceder de América Latina, vivir en condiciones precarias o ser transfundido con unidades que contengan cualquier forma del parásito ${ }^{(8,10)}$. El estado inmunológico de los pacientes es un factor determinante en el curso de la enfermedad, ya que este parásito puede llegar a comportarse como un agente oportunista de alta agresividad en individuos con inmunodeficiencias, causando cuadros clínicos como meningoencefalitis, miocarditis o, en casos muy severos, fallas multisistémicas ${ }^{(11)}$.

La quimioterapia, adicional a un control oportuno de vectores y rigurosidad en el control de las transfusiones, son los elementos más importantes para el manejo de esta enfermedad. No obstante, en la actualidad no se cuenta con un amplio grupo de medicamentos y estrategias para prevenir y controlar la tripanosomiasis americana; el mercado farmacéutico solo ofrece actualmente nifurtimox y benznidazol como opciones terapéuticas ${ }^{(1,2)}$. Estos dos medicamentos cuentan con inconvenientes, como el desarrollo de toxicidad, la paulatina aparición de resistencia producto de la variación antigénica, poca efectividad en el tratamiento de fases crónicas de la enfermedad, requerimientos de administración parenteral y larga duración de los esquemas de tratamiento ${ }^{(3,5,5,12)}$.

En el último siglo se ha trabajado ampliamente en el desarrollo de un tratamiento efectivo y con menos inconvenientes para esta parasitosis. Sin embargo, en los últimos 40 años no ha cambiado en forma considerable el tratamiento disponible. Se han obtenido resultados satisfactorios con el uso de estos medicamentos en fases agudas de la enfermedad, tripanosomiasis congénita y accidentes de laboratorio. Pero, la efectividad en las fases crónicas de la enfermedad es notoriamente menor ${ }^{(13)}$.

\section{MANEJO ACTUAL DE LA TRIPANOSOMIASIS AMERICANA}

Como se mencionó anteriormente, para el tratamiento de la tripanosomiasis americana solo se dispone actualmente de dos medicamentos: benznidazol y nifurtimox. De estas opciones terapéuticas, la que se usa con mayor frecuencia es benznidazol, ya que se ha demostrado que es mejor tolerado por los pacientes ${ }^{(14-16)}$.

Se recomienda que todas las personas que hayan sido diagnosticadas con infección aguda o infección congénita por Trypanosoma cruzi, aquellas que tengan sistemas inmunitarios deprimidos y todos los niños con infección crónica deben recibir tratamiento antiparasitario con alguno de estos dos fármacos disponibles. Los adultos con infección crónica también se pueden beneficiar del tratamiento, pero aún existen muchas controversias en cuanto a su efectividad; se considera que el tratamiento es opcional para los individuos mayores de 50 años ${ }^{(17)}$.

El esquema farmacológico propuesto en la actualidad para el tratamiento con nifurtimox consiste en administrar el medicamento de forma prolongada, debido a sus propiedades farmacológicas de rápida depuración y baja potencia tripanocida: para adultos, 8 a $10 \mathrm{mg}$ por kilogramo de peso por día, vía oral, en 3 o 4 dosis, por un período de 3 a 4 meses; mientras que los jóvenes deben recibir de 12,5 a $15 \mathrm{mg}$ y, los niños menores de 10 años, 15 a $20 \mathrm{mg}$ por kilogramo de peso por día, de la misma forma que los adultos y por el mismo período de tiempo. Por su parte, el tratamiento con benznidazol en los adultos consiste en 5 a $7 \mathrm{mg}$ por kilogramo de peso por día, vía oral en dos dosis, por un periodo de 2 meses, mientras que en los menores de 12 años se administran de 5 a $10 \mathrm{mg}$ por kilogramo al día ${ }^{(3,11,17)}$.

Estos dos medicamentos son compuestos nitrogenados heterocíclicos. El benznidazol es un nitroimidazol, mientras que el nifurtimox es un derivado de nitrofuranos que actúa reduciendo el grupo nitro para originar peróxido de hidrógeno o anión superóxido. Estos compuestos oxidativos son producidos gracias a la enzima superóxido dismutasa, que es la responsable de la reducción de los grupos nitro; estos radicales en presencia de hierro forman un fuerte radical hidroxilo oxidante e incrementan la producción de especies reactivas de oxígeno, que finalmente van a llevar a un estrés oxidativo en el parásito. No obstante, el efecto oxidativo nocivo de estos compuestos resultantes no se limita al parásito, sino que afecta en igual proporción a las células de los mamíferos, lo que lleva al desarrollo de los efectos adversos de estos medicamentos ${ }^{(12,18)}$.

En general, el mecanismo de acción de los medicamentos azoles consiste en bloquear la síntesis del ergosterol -compuesto esencial para la supervivencia del parásito- y otras macromoléculas importantes por medio de enlaces covalentes u otras interacciones entre intermediarios nitroreductores y otros compuestos celulares. Se ha evidenciado adicionalmente que el tratamiento antiparasitario combinado aumenta la efectividad, contribuye a la disminución en las tasas de desarrollo de resistencia y reduce a su vez la toxicidad $^{(19,20)}$.

Algunos autores discuten la efectividad del tratamiento actualmente disponible, sugiriendo que dicha efectividad está determinada por diferentes facto- 
res como el estadio de la enfermedad, la dosis del medicamento, y la edad y procedencia geográfica del paciente que ha sido diagnosticado con esta parasitosis ${ }^{(9,21)}$. En la mayoría de los casos la detección de la enfermedad tiene lugar en las fases crónicas, cuando las secuelas ya mencionadas comienzan a ser más evidentes y el tratamiento antiparasitario parece no ser tan efectivo ${ }^{(14,22)}$.

Los tratamientos antitripanocidas han demostrado en algunos estudios una efectividad cercana al 100\%; como el caso de un seguimiento realizado a un grupo de 66 pacientes, de los cuales 29 fueron sometidos a tratamiento con benznidazol y nifurtimox, en donde se obtuvo un xenodiagnóstico negativo después de un período de 15 años en 93,1\% de los pacientes tratados ${ }^{(22)}$; sin embargo, los resultados de otros estudios no son tan alentadores, pues demuestran bajas tasas de curación, tanto en animales como en humanos ${ }^{(9)}$.

A pesar de la aparente erradicación del parásito, los pacientes deben estar sujetos a un seguimiento periódico con el fin de detectar tempranamente las secuelas crónicas de esta condición ${ }^{(22)}$. Se ha sugerido que los resultados negativos de las pruebas de xenodiagnóstico, hemocultivos o microscopia no se correlacionan de forma definitiva con la eliminación del parásito y la curación de la enfermedad, por lo que se necesita de más estudios para determinar con certeza la efectividad del tratamiento con estos compuestos ${ }^{(23)}$.

Debido a fenómenos sociales y económicos, la tripanosomiasis ha migrado en forma considerable a zonas no endémicas como Norteamérica, Canadá y Europa, haciendo que los pacientes crónicos sean mal diagnosticados y confundidos con otras enfermedades que llevan a inmunosupresión, lo que impide el tratamiento oportuno para esta condición ${ }^{(10,17,24)}$.

Diversas investigaciones han demostrado que la tasa de curación de la tripanosomiasis americana disminuye con la cronicidad de la enfermedad, siendo peores los desenlaces mientras más crónica sea la parasitosis; incluso se ha estimado que tan solo $5,9 \%$ de los pacientes que reciben tratamiento en estadios crónicos alcanza la cura parasitológica ${ }^{(25)}$. Adicionalmente, se ha demostrado cierto grado de resistencia por parte de este parásito a los tratamientos disponibles, condición que podría explicar las bajas tasas de cura de los pacientes ${ }^{(9,26)}$.

A pesar de la poca efectividad con que cuentan estos medicamentos en fases crónicas de la parasitosis, se ha demostrado que el uso de benznidazol en pacientes en estadios avanzados detiene la progresión de las lesiones cardiacas, como la miocardiopatía infecciosa y el estado protrombótico asociado a la presencia del parásito en la sangre, haciendo de este tratamiento la mejor opción disponible actualmente para el tratamiento de la tripanosomiasis americana en cualquier fase ${ }^{(15,24,27)}$.

Es por estos motivos que los estudios que se están llevando a cabo en la actualidad pretenden el desarrollo de un nuevo tratamiento que sea más seguro y efectivo, y que abra las posibilidades que por más de 40 años han sido reducidas, en lo que al tratamiento de la enfermedad de Chagas se refiere ${ }^{(28)}$. Estudios recientes han analizado la toxicidad de estos compuestos antiparasitarios en líneas celulares de mamíferos cultivadas in vitro, y han encontrado que ciertas modificaciones en la estructura de los compuestos derivados del benzimidazol pueden detener selectivamente las infecciones parasitarias, sin productos significativos responsables de toxicidad para el huésped ${ }^{(29)}$.

\section{EFECTOS ADVERSOS DEL TRATAMIENTO}

Según los estudios, se ha evidenciado la aparición de efectos adversos hasta en el 50\% de los pacientes que son manejados con benznidazol, siendo los más frecuentes la dermatitis asociada a hipersensibilidad (no relacionada con la dosis del medicamento ni con la erradicación del parásito), la intolerancia gastrointestinal y la polineuropatía (comprometiendo sistema nervioso central y periférico), los cuales se presentan comúnmente de forma simultánea ${ }^{(30-32)}$. Es imperativo tener en cuenta que la aparición de estos eventos adversos disminuye la adherencia al tratamiento, alcanzando tasas de deserción hasta del $18 \%{ }^{(30)}$.

El benznidazol es un compuesto poco hidrosoluble, condición que afecta directamente su biodisponibilidad. Se han analizado varias estrategias para solucionar este inconveniente, pues este aspecto farmacocinético incide directamente en las dosis que deben ser administradas y, consecuentemente, con la adherencia al tratamiento y la aparición de efectos adversos dependientes de la dosis ${ }^{(33)}$.

Un estudio llevado a cabo en Murcia, España, con 26 pacientes adultos que fueron diagnosticados con enfermedad de Chagas en fase crónica y que completaron el esquema de tratamiento con nifurtimox, demostró la presencia de efectos adversos en $61,5 \%$ de los casos, de los cuales, cerca de $27 \%$ presentó dos o más efectos desfavorables. El evento adverso más frecuente fue la intolerancia a nivel gastrointestinal y anorexia; no obstante, la aparición de estos eventos no llevó a la interrupción del tratamiento ${ }^{(16)}$.

Debido a las implicaciones que tiene el tratamiento de esta parasitosis, es necesario hacer un seguimiento muy estricto de los pacientes, con el fin de evitar las complicaciones de la fase crónica. Se ha evaluado el estado inmunológico de algunos pacientes durante su tratamiento con benznidazol, mostrando en general un perfil de citoquinas regulado a la baja, tanto en inmunidad innata como en inmunidad adquirida, lo que demuestra el efecto inmunomodulador del benznidazol. Estas citoquinas pro-inflamatorias tienen un papel fundamental en la aparición de las complicaciones de la parasitosis, tales como la miocarditis ${ }^{(34,35)}$. 


\section{NUEVOS AVANCES EN EL TRATAMIENTO}

La necesidad de un tratamiento tripanocida efectivo en cualquier fase de la enfermedad, además de la eliminación de los efectos adversos y la reducción de los costos, se hace cada vez más urgente con el paso del tiempo. Es por esto que la comunidad científica está sumando esfuerzos con el objetivo de diseñar un tratamiento costo-efectivo y seguro. Para tal fin se están llevando a cabo investigaciones con moléculas nuevas que han mostrado cierta capacidad tripanocida, incluyendo moléculas derivadas de compuestos naturales, medicamentos que ya han sido utilizados contra otros protozoos e inclusive medicamentos antituberculosos ${ }^{(36-38)}$.

La selectividad de los agentes tripanocidas es un factor que determina la aparición de efectos adversos. Trabajos recientes han experimentado con un compuesto llamado paullone, que consiste en moléculas híbridas de calcona (cetona aromática presente en varios productos) y que actúa directamente sobre el parásito inhibiendo ciclinas dependientes de quinasas, consiguiendo una mayor selectividad y eliminando efectos adversos en un porcentaje no despreciable ${ }^{(39)}$.

También se ha demostrado actividad tripanocida en un nuevo grupo de medicamentos conocido como NBPMs (por las siglas en inglés de nitrobenzyl phosphoramide mustards), los cuales fueron desarrollados originalmente como agentes quimioterapéuticos para el tratamiento del cáncer y que consisten en un grupo anitrobenzil unido a la fosforamida citotóxica de la mostaza. Estos agentes cuentan con una buena selectividad, pues actúan sobre la enzima nitrorreductasa tipo 1 , que está ausente en la mayoría de los organismos eucariotas ${ }^{(28)}$.

Un estudio demostró que las 5,7-diaril-2,3-dihidro-1,4-diazepinas (diarildiazepinas), moléculas sintetizadas por ciclocondensación a partir de flavonas sustituidas con etilendiamina, poseen cierto grado de actividad contra el
T. cruzi; incluso una de las diarildiazepinas mostró una reducción de la proliferación de epimastigotes (una etapa de los insectos de la familia Trypanosomatidae) significativamente mayor que el benznidazol ${ }^{(37)}$.

En la búsqueda de nuevos agentes antitripanosomiásicos selectivos, con poca o ninguna toxicidad para las células de mamíferos, se han desarrollado once análogos del 1,4-naftoquinona imido-sustituida como una nueva clase de agente contra los tripanosomas ${ }^{(40)}$, y se han buscado nuevos blancos terapéuticos moleculares que no sean compartidos por los hospederos, como lo son las enzimas del sistema antioxidante dependiente de tripanotión (péptido similar al glutatión), presente en los tripanosomátidos y en el T. cruzi; estos nuevos blancos terapéuticos parecen ser la solución para el desarrollo de los nuevos tripanocidas ${ }^{(36)}$. Además, se han llevado a cabo ensayos ELA (siglas en inglés, enzyme linked aptamer) para buscar en roedores murinos infestados con T. cruzi posibles biomarcadores que sirvan para la monitorización y evaluación de los futuros fármacos antitripanosomiásicos ${ }^{(41)}$.

Otros fármacos derivados del indazol también han sido estudiados en la búsqueda de un tratamiento que cumpla con los requerimientos ya mencionados. Su mecanismo de acción consiste en una reducción electroquímica, a partir de la cual se forman radicales nitro-anión ${ }^{(42)}$. Incluso se han realizado ensayos clínicos con azoles antifúngicos como el posaconazol y ravuconazol, los cuales revelaron la inferioridad de estos medicamentos en comparación con el benznidazol ${ }^{(43)}$.

A su vez, se están llevando a cabo estudios con compuestos de origen natural para analizar sus posibles propiedades tripanocidas. Moléculas como los terpenoides han sido postuladas como opciones terapéuticas con menos efectos adversos y más efectividad contra la tripanosomiasis; estos compuestos son fundamentales en algunas especies de plantas como mecanismo de defensa contra herbívoros y microorganismos potencialmente patógenos ${ }^{(44,45)}$.

Entre los productos de origen vegetal se encuentran por ejemplo los derivados de la Eugenia uniflora, una planta tropical conocida comúnmente como cereza de cayena, de la cual se ha demostrado su actividad biológica antimicrobiana y se han aislado algunos de sus fitocomponentes, como los flavonoides de iricitrina, quercitina y quercetrina, además de esteroides y compuestos titerpenoides, entre otros. Otras plantas de origen brasilero también han mostrado actividad antitripanosoma; especies como Ampelozizyphus amazonicus, planta endémica de la región amazónica, ha demostrado tener utilidad profiláctica contra la parasitosis ${ }^{(46)}$. Otras moléculas que están siendo estudiadas son las lactonas sesquiterpenas, que cuentan con diferentes actividades y usos en humanos, entre ellos, anticancerígeno, antiinflamatorio, antimicrobiano y antiprotozoo ${ }^{(47)}$.

Son moléculas que están siendo analizadas como nuevas opciones terapéuticas la benzidrazida y nifuroxazida, que a pesar de que su mecanismo de acción no ha sido dilucidado por completo, se ha demostrado que actúan reduciendo grupos nitro y formando radicales libres tóxicos para el microorganismo invasor (48). Asimismo, se ha evaluado la actividad tripanocida de algunos derivados de tetrahidroquinolinas, compuestos en los que previamente se había evidenciado su efectividad en el tratamiento contra Plasmodium falciparum ${ }^{(49)}$.

Las estructuras celulares que contienen tubulina cumplen funciones fundamentales como la migración cromosómica a los polos de la célula durante la mitosis, transporte celular e incluso el proceso de apoptosis, lo que hace a estas estructuras un blanco apto para el tratamiento de diversas condiciones como el cáncer y las parasitosis humanas; es por esto que estudios recientes han cuestionado acerca de la actividad tripanocida de los agentes antitubulina ${ }^{(50)}$.

Adicionalmente, medicamentos 
como el alopurinol, un análogo de purinas, también ha mostrado actividad en contra de este protozoo, actuando por medio de la interrupción de la síntesis de ARN y proteínas, de la misma forma que algunos medicamentos antifúngicos como el itraconazol ${ }^{(51)}$. Ambos medicamentos fueron evaluados en Chile, en un estudio que estudió la presencia de parásitos y parámetros electrocardiográficos tras 11 años de administrar alguno de estos dos medicamentos a 109 pacientes. De esta forma, demostraron que ambos medicamentos podrían ser una alternativa para el tratamiento de la tripanosomiasis americana crónica en dicho país; aunque estadísticamente los medicamentos fueron similares, los autores refieren que se encontró un mayor beneficio con el itraconazol, debido a sus menores efectos adversos, mayor impacto en las anormalidades electrocardiográficas y mejor cubrimiento de la cepa más común de la región ${ }^{(52)}$.

Otra de las alternativas evaluadas actualmente, son las combinaciones del tratamiento tradicional con antifúngicos, en particular aquellas entre azoles -como posaconazol y ravuconazol- con el benznidazol. Un ejemplo de esto es el ensayo clínico STOP CHAGAS, que evalúa el tratamiento con posaconazol (400 mg b.i.d., por 60 días), benznidazol (200 mg b.i.d. por 60 días) y una combinación de posaconazol $(400 \mathrm{mg}$ b.i.d., por 60 días) con benznidazol (200 mg b.i.d. por 60 días) y su efecto en la presencia de $T$. cruzi en la sangre de pacientes con enfermedad crónica sin compromiso cardiaco. Aún se esperan los resultados de este estudio ${ }^{(53)}$.

\section{CONCLUSIÓN}

A pesar de los ensayos clínicos y demás estudios experimentales que se han llevado a cabo en los últimos años, aún se carece de investigaciones que demuestren realmente la efectividad y seguridad de los medicamentos desarrollados para combatir la tripanosomiasis americana.

Existe un sinnúmero de variables que deben ser tenidas en cuenta a la hora de realizar los estudios e interpretar los resultados que se obtienen de los mismos, tales como el estadio en que se encuentran los pacientes al momento del diagnóstico de la enfermedad de Chagas, las características sociodemográficas de la población de estudio, la dosis de los medicamentos y el tiempo que dura el tratamiento, además de la variabilidad genética de los parásitos aislados, los cuales pueden poseer mayor o menor grado de resistencia a los tripanocidas evaluados ${ }^{(9)}$.

Es por esto que persiste actualmente la controversia en cuanto a los resultados de los estudios reportados en la literatura, y se necesita del desarrollo de otras alternativas que sean más seguras y efectivas, diferentes al mismo esquema que se ha manejado por décadas. Se busca mejorar la calidad de vida y el pronóstico de los miles de pacientes afectados anualmente por esta parasitosis.

\section{REFERENCIAS BIBLIOGRÁFICAS}

1. Alsford S, Kelly JM, Baker N, Horn D. Genetic dissection of drug resistance in trypanosomes. Parasitology. 2013;140(12):1478-91. doi: 10.1017/ So03118201300022X

2. Bahia MT, de Andrade IM, Martins TA, do Nascimento ÁF, Diniz Lde F, Caldas IS, et al. Fexinidazole: a potential new drug candidate for Chagas disease. PLoS Negl Trop Dis. 2012;6(11):e1870. doi: 10.1371/journal.pntd.0001870.

3. Barrett MP, Croft SL. Management of trypanosomiasis and leishmaniasis. Br Med Bull. 2012;104:17596. doi: 10.1093/bmb/lds031.

4. Vega MC, Rolón M, Montero-Torres A, FonsecaBerzal C, Escario JA, Gómez-Barrio A, et al. Synthesis, biological evaluation and chemometric analysis of indazole derivatives. 1,2-disubstituted 5-nitroindazolinones, new prototypes of antichagasic drug. Eur J Med Chem. 2012;58:214-27. doi: 10.1016/j.ejmech.2012.10.009.

5. França da Silva C, Jaen Batista D, Melo G, Mello de Souza E, Ripoll E, Bernardino P, et al. In vitro and in vivo investigation of the efficacy of arylimidamide DB1831 and its mesylated salt form - DB1965 against Trypanosoma cruzi infection. PloS One. 2012;23:1-8. doi: 10.1371/journal.pone.0030356.

6. Pinto AY, Valente C, Coura JR, Valente SA, Junqueira AC, Santos LC, et al. Clinical follow-up of responses to treatment with benznidazol in Amazon: a cohort study of acute Chagas disease. PLoS One. 2013;8(5):e64450. doi: 10.1371/journal. pone.0064450.

7. Barbosa MP, do Carmo AA, Rocha MO, Ribeiro AL. Ventricular arrhythmias in Chagas disease. Rev Soc Bras Med Trop. 2015;48(1):4-10. doi:
10.1590/0037-8682-0003-2014.

8. Moreira da Silva R, Oliveira LT, Silva Barcellos NM, de Souza J, de Lana M. Preclinical monitoring of drug association in experimental chemotherapy of Chagas' disease by a new HPLC-UV method. Antimicrob Agents Chemother. 2012;56(6):3344-8. doi: 10.1128/AAC.05785-11.

9. Oliveira-Silva JC, Machado-de-Assis GF, Oliveira MT, Paiva NC, Araújo MS, Carneiro CM, et al. Experimental benznidazole treatment of Trypanosoma cruzi II strains isolated from children of the Jequitinhonha Valley, Minas Gerais, Brazil, with Chagas disease. Mem Inst Oswaldo Cruz. 2015;110(1):8694. doi: 10.1590/0074-02760140260.

10. Requena-Méndez A, Aldasoro E, de Lazzari E, Sicuri E, Brown M, Moore DA, et al. Prevalence of Chagas disease in Latin-American migrants living in Europe: a systematic review and meta-analysis. PLoS Negl Trop Dis. 2015;9(2):e0003540. doi: 10.1371/journal.pntd.0003540.

11. Benitez R, Noemi I, Tassara R, Catalán P, Avilés $C L$. Prophylaxis against Chagas disease in pediatric and adult patients undergoing solid organ and hematopoietic stem cells transplantation. Rev Chilena Infectol. 2012;29 Suppl 1:S41-3.

12. Boiani M, Piacenza L, Hernández P, Boiani L, Cerecetto $\mathrm{H}$, González M, et al. Mode of action of nifurtimox and $\mathrm{N}$-oxide-containing heterocycles against Trypanosoma cruzi: is oxidative stress involved? Biochem Pharmacol. 2010;79(12):173645. doi: 10.1016/j.bcp.2010.02.009.

13. Honorato SB, Mendonça JS, Boechat N, Oliveira AC, Mendes Filho J, Ellena J, et al. Novel polymorphs of the anti-Trypanosoma cruzi drug benznidazole. Spectrochim Acta A Mol Biomol Spectrosc. 2014;118:389-94. doi:10.1016/j.saa.2013.08.096.

14. Fuentes BR, Maturana AM, de la Cruz MR. Efficacy of nifurtimox for the treatment of chronic Chagas disease. Rev Chilena Infectol. 2012;29(1):82-6. doi: 10.4067/S0716-10182012000100013.

15. Cencig S, Coltel N, Truyens C, Carlier Y. Evaluation of benznidazole treatment combined with nifurtimox, posaconazole or AmBisome ${ }^{\circledR}$ in mice infected with Trypanosoma cruzi strains. Int J Antimicrob Agents. 2012;40(6):527-32. doi: 10.1016/j. ijantimicag.2012.08.002.

16. Murcia L, Carrilero B, Segovia M. Limitations of currently available Chagas disease chemotherapy. Rev Esp Quimioter. 2012;25(1):1-3.

17. Bern C, Montgomery SP, Herwaldt BL, Rassi A Jr, Marin-Neto JA, Dantas RO, et al. Evaluation and treatment of Chagas disease in the United States: a systematic review. JAMA. 2007;298(18):2171-81.

18. Bianchi F, Cucunubá Z, Guhl F, González NL, Freilij $\mathrm{H}$, Nicholls RS, et al. Follow-up of an asymptomatic Chagas disease population of children after treatment with nifurtimox (Lampit) in a sylvatic endemic transmission area of Colombia. PLoS Negl Trop Dis. 2015;10(2):e0003465. doi: 10.1371/ journal.pntd.0003465.

19. Diniz Lde F, Urbina JA, de Andrade IM, Mazzeti AL, Martins TA, Caldas IS, et al. Benznidazole and posaconazole in experimental Chagas disease: positive interaction in concomitant and sequential treatments. PLoS Negl Trop Dis. 2013;7(8):e2367.

20. Piaggio E, Sancéau J, Revelli S, Bottasso O, Wietzerbin J, Serra E. Trypanocidal drug benznidazole impairs lipopolysaccharide induction of macrophage nitric oxide synthase gene transcription through inhibition of NF-KB activation. J Immunol. 2001;167:3422-26.

21. Pinazo MJ, Guerrero L, Posada E, Rodriguez E, 
Soy D, Gascon J. Benznidazole-related adverse drug reactions and their relationship to serum drug concentrations in patients with chronic chagas disease. Antimicrob Agents Chemother. 2013;57(1):390-5. doi: 10.1128/AAC.01401-12.

22. Fabbro D, Velazquez E, Bizai ML, Denner S, Olivera $\mathrm{V}$, Arias E, et al. Evaluation of the ELISA-F29 test as an early marker of therapeutic efficacy in adults with chronic Chagas disease. Rev Inst Med Trop Sao Paulo. 2013;55(3). doi: 10.1590/S003646652013000300005.

23. Santamaria C, Chatelain E, Jackson $Y$, Miao Q, Ward BJ, Chappuis F, et al. Serum biomarkers predictive of cure in Chagas disease patients after nifurtimox treatment. BMC Infect Dis. 2014;14:302. doi: 10.1186/1471-2334-14-302.

24. Pinazo MJ, Espinosa G, Cortes-Lletget C, Posada Ede J, Aldasoro E, Oliveira I, Muñoz J, Gállego M, Gascon J. Immunosuppression and Chagas disease: a management challenge. PLoS Negl Trop Dis. 2013;7(1):e1965. doi: 10.1371/journal. pntd.0001965.

25. Matta PM, Gutierrez FR, Nascimento MS, Do-ValleMatta MA, Silva JS. Antiparasitical chemotherapy in Chagas' disease cardiomyopathy: current evidence. Trop Med Int Health. 2012;17(9):1057-65. doi: 10.1111/j.1365-3156.2012.03025.x.

26. Jorge SD, Palace F, Mesquita KF, Ishii M, Ferreira AK, Berra CM, et al. Ligand-based design, synthesis, and experimental evaluation of novel benzofuroxan derivatives as anti-Trypanosoma cruzi agents. Eur J Med Chem. 2013;64:200-14. doi: 10.1016/j.ejmech.2013.03.053.

27. Molina A, Campos C, Lapier M, Duaso J, Kemmerling $U$, Galanti N, et al. Benznidazole prevents endothelial damage in an experimental model of Chagas disease. Acta Trop. 2013;127(1):6-13. doi: 10.1016/ j.actatropica.2013.03.006.

28. Buchanan-Kilbey G, Djumpah J, Papadopoulou MV, Bloomer W, Hu L, Wilkinson SR, et al. Evaluating the developmental toxicity of trypanocidal nitroaromatic compounds on zebrafish. Acta Trop. 2013;128(3):701-5. doi: 10.1016/j.actatropica.2013.07.022.

29. Diaz-Chiguer DL, Márquez-Navarro A, NoguedaTorres B, de la Luz León-Ávila G, Pérez-Villanueva $J$, Hernández-Campos A, et al. In vitro and in vivo trypanocidal activity of some benzimidazole derivatives against two strains of Trypanosoma cruzi. Acta Trop. 2012;122(1):108-12. doi: 10.1016/j. actatropica.2011.12.009.

30. Hasslocher AM, do Brasil PE, de Sousa AS, Xavier SS, Chambela MC, Sperandio da Silva GM. Safety of benznidazole use in the treatment of chronic Chagas' disease. J Antimicrob Chemother. 2012;67(5):1261-6. doi: 10.1093/jac/dks027.

31. Pérez JA, Sojo J, Norman F, Monge B, Díaz M, Albajar $P$, et al. Nifurtimox therapy for Chagas disease does not cause hypersensitivity reactions in patients with such previous adverse reactions during benznidazole treatment. Acta Trop. 2013;127(2):101-4. doi: 10.1016/j.actatropica. 2013.04.003.

32. Tornheim JA, Lozano DF, Gilman RH, Castellon M, Solano MA, Sullca W, et al. Improved completion rates and characterization of drug reactions with an intensive Chagas disease treatment program in rural Bolivia. PLoS Negl Trop Dis. 2013;7(9): e2407. doi: 10.1371/journal.pntd.0002407.
33. Leonardi D, Bombardiere ME, Salomon CJ. Effects of benznidazole: cyclodextrin complexes on the drug bioavailability upon oral administration to rats. Int J Biol Macromol. 2013;62:543-8. doi: 10.1016/j. ijbiomac.2013.10.007.

34. Sathler R, Marquete D, Elói SM, Dias E, Teixeira A, Martins OA. Blood leukocytes from benznidazoletreated indeterminate chagas disease patients display an overall type-1-modulated cytokine profile upon short-term in vitro stimulation with trypanosoma cruzi antigens. BMC Infect Dis. 2012;12(123). doi: 10.1186/1471-2334-12-123.

35. Wen JJ, Garg NJ. Proteome expression and carbonylation changes during Trypanosoma cruzi infection and Chagas disease in rats. Mol Cell Proteomics. 2012;11(4):M111.010918. doi: 10.1074/ mcp.M111.010918.

36. González-Chávez Z, Olin-Sandoval V, RodiguezZavala JS, Moreno-Sánchez R, Saavedra E. Metabolic control analysis of the Trypanosoma cruzi peroxide detoxification pathway identifies tryparedoxin as a suitable drug target. Biochim Biophys Acta. 2015;1850(2):263-73. doi: 10.1016/j. bbagen. 2014.10.029.

37. Menezes JC, Beatriz L, de Abreu PM, da Silva K, Martins C, Taylor JG. Synthesis and anti-Trypanosoma cruzi activity of diaryldiazepines. Molecules. 2015;20:43-51. doi:10.3390/molecules20010043.

38. Upadhayaya RS, Dixit SS, Földesi A, Chattopadhyaya J. New antiprotozoal agents: their synthesis and biological evaluations. Bioorg Med Chem Lett. 2013;23(9):2750-8. doi: 10.1016/j. bmcl.2013.02.054.

39. Ryczak J, Papini M, Lader A, Nasereddin A, Kopelyanskiy D, Preu L, et al. 2-Arylpaullones are selective antitrypanosomal agents. Eur J Med Chem. 2013;64:396-400. doi: 10.1016/j. ejmech.2013.03.065.

40. Khraiwesh MH, Lee CM, Brandy Y, Akinboye ES, Berhe S, Gittens G, et al. Antitrypanosomal activities and cytotoxicity of some novel imidosubstituted 1,4-naphthoquinone derivatives. Arch Pharm Res. 2012;35(1):27-33. doi: 10.1007/ s12272-012-0103-1.

41. de Araujo FF, Nagarkatti R, Gupta C, Marino AP, Debrabant A. Aptamer-based detection of disease biomarkers in mouse models for Chagas drug discovery. PLoS Negl Trop Dis. 2015;9(1):e3451. doi: 10.1371/journal.pntd.0003451.

42. Diaz CA, Olea CA, Zapata GA, Lapier M, Mura F, Aguilera B, et al. Biological and chemical study of fused tri- and tetracyclic indazoles and analogues with important antiparasitic activity. Spectrochim Acta A Mol Biomol Spectrosc. 2012;95:670-8. doi: 10.1016/j.saa.2012.04.076.

43. Keenan M, Chaplin JH. A new era for chagas disease drug discovery? Prog Med Chem. 2015;54:185230. doi: 10.1016/bs.pmch.2014.12.001.

44. Ramirez I, Marin C, Chahboun R, Messouri I, Olmo F, Rosales MJ, et al. In vitro and in vivo studies of the trypanocidal activity of four terpenoid derivatives against Trypanosoma cruzi. Am J Trop Med Hyg. 2012;87(3):481-8. doi: 10.4269/ ajtmh.2012.11-0471.

45. Borges AR, Aires JR, Higino TM, de Medeiros Md, Citó AM, Lopes JA, et al. Trypanocidal and cytotoxic activities of essential oils from medicinal plants of Northeast of Brazil. Exp Parasitol. 2012;132(2):123-8. doi: 10.1016/j.exppara.2012.06.003.
46. Santos KK, Matias EF, Tintino SR, Souza CE, Braga MF, Guedes GM, et al. Anti-Trypanosoma cruzi and cytotoxic activities of Eugenia uniflora L. Exp Parasitol. 2012;131(1):130-2. doi: 10.1016/j. exppara.2012.02.019.

47. Cogo J, Caleare AO, Ueda T, Filho BP, Ferreira IC Nakamura CV. Trypanocidal activity of guaianolide obtained from Tanacetum parthenium (L.) SchultzBip and its combinational effect with benznidazole. Phytomedicine. 2012;20(1):59-66. doi: 10.1016/j. phymed.2012.09.011.

48. Palace F, Jorge SD, Pasqualoto KF, Ferreira AK, Maria DA, Zorzi RR, et al. 5-Nitro-2-furfuriliden derivatives as potential anti-Trypanosoma cruzi agents: design, synthesis, bioactivity evaluation, cytotoxicity and exploratory data analysis. Bioorg Med Chem. 2013;21(17):5395-406. doi: 10.1016/j. bmc.2013.06.017.

49. Fonseca $C$, Merchán DR, Romero AR, Escario JA, Kouznetsov VV, Gómez A. Selective activity of 2,4-diaryl-1,2,3,4 tetrahydroquinolines on Trypanosoma cruzi epimastigotes and amastigotes expressing $\beta$-galactosidase. Bioorg Med Chem Lett. 2013;23(17):4851-6. doi: 10.1016/j. bmcl.2013.06.079.

50. Lama R, Sandhu R, Zhong B, Li B, Su B. Identification of selective tubulin inhibitors as potential anti-trypanosomal agents. Bioorg Med Chem Lett. 2012;22(17):5508-16. doi: 10.1016/j. bmcl.2012.07.023.

51. Argüello RJ, Albareda MC, Alvarez MG, Bertocchi G, Armenti AH, Vigliano C, et al. Inhibitory receptors are expressed by Trypanosoma cruzi-specific effector T cells and in hearts of subjects with chronic Chagas disease. PLoS One. 2012;7(5):e35966. doi: 10.1371/journal.pone.0035966.

52. Apt W, Arribada A, Zulantay I, Solari A, Sánchez G, Mundaca K, et al. Itraconazole or allopurinol in the treatment of chronic American trypanosomiasis: the results of clinical and parasitological examinations 11 years post-treatment. Ann Trop Med Parasitol. 2005;99(8):733-41. PubMed PMID: 16297286.

53. Urbina JA. Recent clinical trials for the etiological treatment of chronic Chagas disease: advances, challenges and perspectives. J Eukaryot Microbiol. 2015;62(1):149-56. doi: 10.1111/jeu.12184.

Artículo recibido el 23 de julio de 2015 y aceptado para publicación el 4 de noviembre de 2015.

Declaración de originalidad del artículo: los autores garantizan que el presente artículo no ha sido publicado, total ni parcialmente, en otra revista cientifica.

Fuente de financiamiento: Ninguna.

Declaración de conflictos de interés: los autores declaran que no poseen ningún conflicto de interés.

\section{Correspondencia:}

Daniel Gallego

Dirección: calle 78B No. 72A-109, Medellín, Colombia Teléfono: +57(4) 4936300

Correo electrónico: daniel.gallegogo@upb.edu.com 